\title{
Behavioural Assessment of the Dysexecutive Syndrome (BADS): Adaptação e Evidências de Validade
}

\author{
Greici Rössler Macuglia - Universidade Federal do Rio Grande do Sul, Porto Alegre, Brasil \\ Rosa Maria Martins de Almeida - Universidade Federal do Rio Grande do Sul, Porto Alegre, Brasil \\ Fabiane Caillava Santos - Universidade Federal do Rio Grande do Sul, Porto Alegre, Brasil \\ Cláudia Hofheinz Giacomoni - Universidade Federal do Rio Grande do Sul, Porto Alegre, Brasil
}

\begin{abstract}
Resumo
As funções executivas são habilidades que permitem um indivíduo direcionar seu funcionamento cognitivo, comportamental e emocional a metas. Para avaliá-las existem poucos instrumentos ecologicamente válidos. Para superar essa limitação, foi desenvolvida a Behavioural Assessment of the Dysexecutive Syndrome (BADS). O objetivo deste estudo foi adaptar e investigar evidências de validade de conteúdo da BADS para o contexto brasileiro. Foram realizadas três traduções do instrumento, sintetizadas em uma única versão e encaminhada a três juízes. Posteriormente, a BADS foi submetida a um grupo focal (5 adultos saudáveis) e estudo-piloto (amostra clínica com 40 pacientes neurológicos, portadores de Parkinson). O cálculo do coeficiente de validade de conteúdo total foi considerado aceitável $(>0,80)$; e a média do coeficiente kappa para os subtestes foi de 0,55, e para o questionário foi de 0,75 , moderado e sólido, respectivamente. Os dados sugerem que a BADS está apta a ser utilizada em novos estudos para a investigação de outras propriedades psicométricas, como validade de construto, fidedignidade e normatização. Palavras-chave: funções executivas, síndrome disexecutiva, validade
\end{abstract}

\section{Behavioral Assessment of the Dysexecutive Syndrome (BADS): Adaptation and Validity Evidence}

\begin{abstract}
Executive functions are skills that allow an individual to direct their cognitive, behavioral and emotional functioning to goal achievement. In order to evaluate them, there are few ecologically valid instruments. Behavioral Assessment of the Dysexecutive Syndrome (BADS) was developed to overcome this limitation. The objective of this study was to adapt and investigate BADS content validity for the Brazilian context. Three translations of the instrument from English into Portuguese were done, and were later synthesized into a single version to be sent to three judges. Subsequently, BADS was applied to a focus group (5 healthy adults) and a pilot study (40 clinical neurological patients, with Parkinson's Disease). The calculation of the total content validity coefficient was considered acceptable $(>0.80)$; and the average coefficient kappa subtests was 0.55 and the survey was 0.75 , moderate and solid, respectively. The data suggest that BADS can be used in further studies to investigate other psychometric properties as construct validity, reliability and standardization.
\end{abstract}

Keywords: executive functions, dysexecutive syndrome, validity

Behavioural Assessment of the Dysexecutive Syndrome (BADS): Adaptación y Evidencias de Validez

\section{Resumen}

Las funciones ejecutivas son habilidades que permiten a los individuos direccionar su funcionamiento cognitivo, conductual y emocional, hacia sus metas. Para evaluarlas existen pocos instrumentos ecológicamente válidos. Para superar esta limitación, fue desarrollada la Behavioural Assessment of the Dysexecutive Syndrome (BADS). El objetivo de este estudio fue adaptar e investigar evidencias de validez del contenido de la BADS para el contexto brasileño. Se realizaron tres traducciones del instrumento, sintetizadas en una sola versión y encaminada a tres jueces. Posteriormente, la BADS fue sometida a un grupo de enfoque (5 adultos sanos) y a un estudio piloto (muestra clínica de 40 pacientes neurológicos, con Parkinson). El cálculo del coeficiente de validez de contenido total fue considerado aceptable $(>0.80)$; y la media del coeficiente kappa para los sub-tests fue de 0.55 y para el cuestionario fue de 0.75 , moderado y fuerte, respectivamente. Los datos sugieren que la BADS puede ser utilizada en nuevos estudios para la investigación de otras propiedades psicométricas, como validez del constructo, confiabilidad y baremación.

Palabras clave: funciones ejecutivas, síndrome disejecutivo, validez

As funções executivas (FE) são habilidades complexas que resultam, entre outras estruturas, da atividade desempenhada pelas áreas pré-frontais (Galhardo, Amaral, \& Vieira, 2009) e permitem a um indivíduo direcionar seu funcionamento cognitivo, comportamental e emocional a metas. Para tanto, são necessárias, entre outras competências, a flexibilidade cognitiva, resolução de problemas, planejamento, controle inibitório e julgamento temporal (Hamdan \& Pereira, 2009). O prejuízo em diversas dessas funções é denominado de síndrome disexecutiva (SD), comum em diversos quadros psiquiátricos e neurológicos, necessitando de avaliação adequada para a organização do devido 
programa de reabilitação (Borges et al., 2010; Godefroy, Garcia, Bugnicourt, Leclercq, \& Roussel, 2014).

Para dar conta da complexidade das FEs, é necessária a inclusão de uma ampla gama de instrumentos para a avaliação neuropsicológica desse domínio. Esses instrumentos podem estar agrupados em baterias formais desenvolvidas especificamente para a avaliação de tais funções, como é o caso da BADS, ou em baterias flexíveis, em que o examinador define os critérios para inclusão e agrupamento deles (Malloy-Diniz, Paula, Loschiavo-Alvares, Fuentes, \& Leite, 2010).

O instrumento considerado padrão ouro para avaliar as FE é o Teste Wisconsin de Classificação de Cartas (WCST), desenvolvido para a identificação do domínio flexibilidade mental na resolução de problemas, ou seja, a capacidade de o indivíduo raciocinar abstratamente e modificar suas estratégias cognitivas como resposta a alterações nas contingências ambientais (Strauss, Sherman, \& Spreen, 2006). Além deste, o Teste das Trilhas (Montiel \& Seabra, 2012) e a Torre de Londres (Shallice, 1982) têm demonstrado sensibilidade na avaliação dos mesmos domínios executivos (Perfetti et al., 2010). No entanto, os instrumentos tradicionais apresentam algumas limitações, pois alguns pacientes podem apresentar um bom desempenho sobre essas medidas, mas demonstrar sintomas evidentes de alta desorganização do comportamento cotidiano, uma vez que essas avaliações não possuem relação com o comportamento da pessoa testada em situações naturais, isto é, não apresentam resultados ecologicamente válidos (Hamdan \& Pereira, 2009; Parsons, 2011).

Além disso, a avaliação das FEs não deve basear-se apenas na testagem neuropsicológica do paciente. Os testes fornecem informações objetivas sobre o nível de comprometimento dessas funções, no entanto, dados importantes sobre a extensão e o impacto dos prejuízos também são obtidos por meio da entrevista clínica, observação comportamental e escalas de avaliação. Para tanto, familiares e cuidadores são figuras importantes para a complementação das informações, uma vez que, a falta de insight e prejuízos na autoconsciência pode ocorrer nesses pacientes (Malloy-Diniz et al., 2010).

$\mathrm{Na}$ tentativa de superar essas limitações dos instrumentos tradicionais foi desenvolvida a bateria neuropsicológica chamada Behavioural Assessment of the Dysexecutive Syndrome (BADS) (Wilson, Alderman, Burgess, Emslie, \& Evans, 1996). Esse instrumento foi projetado para predizer problemas cotidianos decorrentes da SD. Sua criação foi influenciada por dois modelos teóricos. O primeiro deles é o Modelo de Memória de Trabalho que hipotetiza a existência de um executivo central, concebido como um controlador geral, organizador, planejador e alocador de recursos (Baddeley \& Hitch, 1974). O segundo é o Modelo Atencional Supervisor constituído por dois mecanismos de atenção: um sistema de programação e contenção responsável pelos aspectos mais rotineiros de atenção, e um sistema supervisor atencional responsável pelos níveis mais elevados, envolvido em tarefas que exigem mais dessa função (Shallice, 1982).

A BADS compreende seis subtestes, que avaliam FE, tais como a flexibilidade cognitiva (Shift Cards); resolução de problemas (Action Program); planejamento e controle inibitório (Key Search, Zoo Map, Modified Six Elements); julgamento temporal (Temporal Judgement). Além dos subtestes, a bateria apresenta dois questionários (Dysexecutive Questionnaire - DEX): um a ser respondidos pelo paciente e outro por um familiar ou cuidador.

A flexibilidade cognitiva, como mencionado acima, consiste na habilidade de alternar a direção das ações e pensamentos de acordo com as exigências impostas pelo ambiente, modificando-as se necessárias. A inflexibilidade cognitiva pode se apresentar por meio de comportamentos perseverativos e estereotipados (Malloy-Diniz, Sedo, Fuentes, \& Leite, 2008). A resolução de problemas corresponde a um conjunto de procedimentos adotados objetivando a eliminação das barreiras encontradas entre o estado presente e o que se busca atingir. Esse processo envolve um ciclo de etapas sucessivas necessárias para se atingir a resolução de um problema (Sternberg, 2000).

Planejamento é a capacidade de estabelecer a maneira mais efetiva de atingir um objetivo, considerando os passos a serem tomados e os instrumentos necessários (Fuentes, Malloy-Diniz, Camargo, \& Consenza, 2014). Em pessoas nas quais há prejuízo no planejamento, costumam resolver seus problemas por meio do método de tentativa e erro (Malloy-Diniz et al., 2008).

O controle inibitório está relacionado à capacidade de inibir respostas, sejam elas respostas prevalentes, respostas a estímulos distratores ou respostas já em curso. A impulsividade está relacionada a dificuldades no controle inibitório (Barkley, 2001).

Já o julgamento temporal permite antecipar eventos relevantes para a obtenção dos objetivos de uma tarefa. Esse domínio possibilita compreender a importância do direcionamento da atenção no tempo para 
organizar a interação e adaptação do indivíduo ao meio (Silva, Macedo, Mariani, Araújo, \& Carreiro, 2011).

Esses domínios das FEs se encontram comprometidos em muitas patologias neurológicas, entre elas a doença de Parkinson (DP), uma condição neurodegenerativa progressiva caracterizada por quatro sinais cardinais: tremor, rigidez, bradicinesia e instabilidade postural (Imamura, Wada-Isoe, Kitayama, \& Nakashima, 2008; Kummer \& Teixeira, 2009). Por muitos anos, a DP foi descrita como sendo uma desordem basicamente motora, negligenciando-se as alterações cognitivas e mentais associadas à patologia (Kamei, Morita, Serizawa, Mizutani, \& Hirayanagi, 2010). No entanto, alterações cognitivas são uma importante causa de incapacidade funcional nesses pacientes, com uma prevalência que pode chegar a $93 \%$, podendo ser tão limitantes quanto os sinais motores (Sammer, Reuter, Hullmann, Kaps, \& Vaitl, 2006; Campos-Sousa, Campos-Sousa, Ataíde Jr., Soares, \& Almeida, 2010). Entre os prejuízos cognitivos mais comuns na DP estão as alterações das FEs, que podem estar presentes desde os primeiros estágios da doença (Mckinlay, Grace, Dalrymple-Alford, \& Roger, 2010).

Dessa forma, utilizar a BADS em amostras de pacientes com DP permitirá conhecer algumas das propriedades psicométricas do instrumento, uma vez que a SD é marcante nessa patologia. Apesar de inúmeras pesquisas no exterior apontando índices psicométricos confiáveis, o instrumento ainda não possui tradução para o português do Brasil, e é consenso entre psicometristas a importância da adaptação de instrumentos para outras culturas. Contudo, além da tradução e adaptação, um instrumento necessita também se submeter a outras avaliações quanto às suas propriedades psicométricas, e entre estas se destaca a validade de conteúdo.

A validade de conteúdo está relacionada à representatividade de um universo de comportamentos (domínio) (Pasquali, 2001) e se refere ao grau em que um instrumento evidencia um domínio específico de conteúdo do que pretende medir (Sampieri, 1996). Estudos dessa natureza investigam a clareza, a representatividade e a relevância dos itens. Para a validação de conteúdo, pode-se utilizar o Coeficiente de Validade de Conteúdo (CVC), e o coeficiente kappa. Ambas medem a porcentagem de juízes que estão em concordância sobre os itens de um instrumento, no entanto, a última é aplicável quando os dados são categóricos (Cassep-Borges, Balbinotti, \& Teodoro, 2010).

Ao longo dos anos, desde o lançamento da BADS, inúmeras pesquisas foram lançadas comprovando suas propriedades psicométricas. Estudos têm mostrado boa validade concorrente da BADS na avaliação de diversas populações neurológicas e psiquiátricas (Norris \& Tate, 2000; Katz, Tadmor, Felzen, \& Hartman-Maeir, 2007), e maior poder discriminativo do instrumento em predizer alterações executivas na vida diária (Burgess, Costello, \& Shallice, 2000; Chaytor \& Schmitter-Edgecombe, 2003).

Dessa forma, corrobora-se a necessidade de estudos que garantam uma adequada adaptação e identifiquem as propriedades psicométricas da BADS para o Brasil, a fim de oferecer aos profissionais da área neuropsicológica um instrumento adequado para avaliar as FEs, habilidades essenciais para a adaptação do indivíduo às atividades do dia a dia, para o desenvolvimento de novas habilidades e o convívio em sociedade. Compreender o funcionamento dessas funções altamente complexas é fundamental para o desenvolvimento de estratégias de intervenção e, para tanto, são necessários instrumentos de avaliação confiáveis, que levem em consideração o contexto ecológico do paciente. Sendo assim, o presente estudo tem como objetivo traduzir, adaptar e investigar evidências de validade de conteúdo da BADS para o contexto brasileiro.

\section{Método}

Existem vários procedimentos possíveis para a realização do processo de tradução e adaptação de um instrumento. No presente estudo, foram utilizadas orientações de autores, como Beaton, Bombardier, Guillemin e Ferraz (2000), Cassepp-Borges, Balbinotti e Teodoro (2010), Geisinger (1994) e de Sandoval e Durán (1998).

Além disso, o adequado processo de adaptação transcultural de um instrumento deve passar por distintas etapas antes que esteja pronto para ser utilizado. A Figura 1 apresenta um esquema utilizado no estudo para os processos de tradução e adaptação da BADS.

\section{Participantes}

O presente estudo foi dividido em sete etapas, resultando em um total de 236 participantes. $\mathrm{Na}$ primeira etapa, ocorreu o processo de tradução, realizado por três avaliadores independentes, entre os quais, dois alunos de pós-graduação proficientes em inglês e um profissional graduado em Letras/Inglês. A seguir, ocorreu a síntese das traduções realizadas pelos mesmos alunos envolvidos na etapa anterior. Na terceira etapa, um comitê formado por três juízes com pós-graduação 


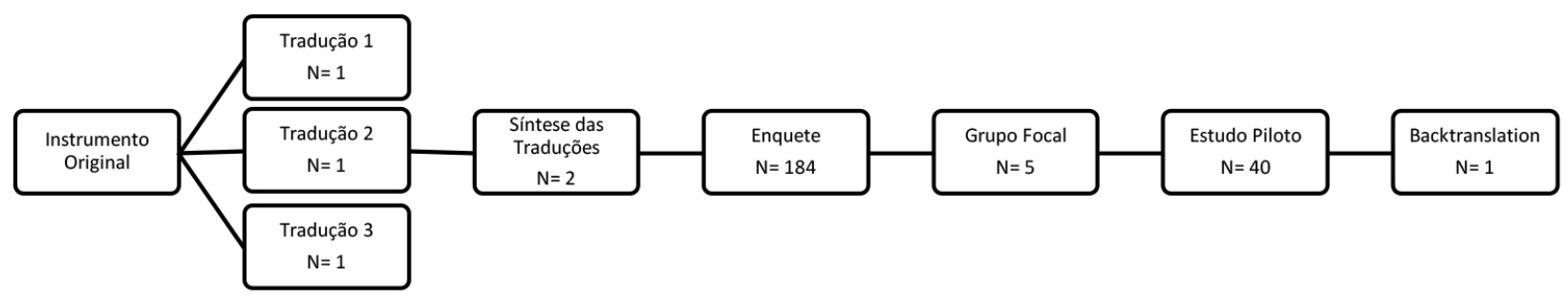

Figura 1. Procedimentos para a adaptação transcultural da BADS.

e ampla experiência em neuropsicologia ficaram responsáveis pela avaliação de conteúdo do instrumento. A quarta etapa avaliou alunos de graduação e pós-graduação da Universidade Federal do Rio Grande do Sul $(N=184)$, objetivando encontrar respostas mais adequadas ao contexto cultural brasileiro para as quatro questões do teste Julgamento Temporal.

A seguir, ocorreu o processo de adaptação, partindo de um grupo focal composto por cinco adultos saudáveis (Tabela 1) e um estudo-piloto formado por uma amostra de conveniência com 40 pacientes com DP, do Ambulatório de Distúrbios do Movimento do Hospital de Clínicas de Porto Alegre-RS, das Estratégias de Saúde da Família e clínicas privadas de Cruz Alta-RS, devidamente diagnosticados por um médico neurologista, com idades entre 44 e 75 anos $(M=$ $63,33 ; D P=8,44)$, sendo $57,5 \%$ do sexo feminino e $72,5 \%$ de quatro a oito anos de escolaridade, caracterizando a quinta e sexta etapa, respectivamente. Por fim, na sétima etapa, ocorreu a retrotradução (backtranslation) da BADS.

\section{Instrumentos}

A BADS (Wilson et al., 1996) é uma bateria ecológica de testes que avalia FE, como controle inibitório, planejamento, resolução de problemas, flexibilidade cognitiva e julgamento temporal. É composta por seis subtestes com uma pontuação individual de zero (pior desempenho) a quatro pontos (melhor desempenho) e pontuação total de zero a 24 pontos, obtidos a partir da soma individual de cada teste. Essa pontuação pode ser convertida em um escore-padrão, com uma média de 100 e um desvio-padrão de 15, e em um escore-padrão por idade. A partir desse escore, pode-se obter a seguinte classificação geral: comprometido, limítrofe, média baixa, média, média alta, superior e muito superior. Alguns items têm componentes de tempo que contribuem para a pontuação do subteste. O instrumento pode ser utilizado em pessoas com idades entre
Tabela 1

Sexo, Idade e Escolaridade dos Participantes do Grupo Focal e Estudo Piloto

\begin{tabular}{lccc}
\hline & Sexo & Idade & Escolaridade \\
\hline $\begin{array}{l}\text { Grupo } \\
\text { focal }\end{array}$ & Feminino & 29 & 11 anos \\
& Feminino & 55 & 11 anos \\
& Feminino & 79 & 4 anos \\
& Masculino & 60 & 5 anos \\
& Masculino & 32 & 8 anos \\
\hline
\end{tabular}

16 e 87 anos, e o tempo de aplicação dura em média 40 minutos.

A BADS possui ainda um questionário com uma versão para ser respondida pelo paciente e outra por um familiar ou cuidador. A seguir são apresentados os subtestes e o questionário DEX.

1. Teste das Cartas de Alternar Regras - avalia tendências de perseveração e flexibilidade mental. Pede-se aos participantes para que respondam ao estímulo (21 cartas vermelhas ou pretas) de acordo com uma das duas regras apresentadas sucessivamente. $\mathrm{O}$ desempenho é avaliado pela capacidade em responder às alterações e adaptar-se às mudanças nas regras. Diante da primeira regra, os avaliados devem responder "sim" para as cartas vermelhas e "não" para as pretas. $\mathrm{Na}$ segunda regra, devem responder "sim" se duas cartas em sequência são da mesma cor, e "não" se forem de cores diferentes. As regras são deixadas em frente ao testando para evitar influências de restrição de memória.

2. Teste do Programa de Ação - avalia a habilidade em planejar e implementar uma solução para um problema prático. O desempenho é avaliado pelo número de passos completados sem auxílio. Em uma base retangular são apoiados dois tubos transparentes. O tubo maior possui uma tampa removível com um 
pequeno furo central, cheio com dois terços de água. O outro, um tubo fino e transparente comporta uma pequena rolha de cortiça. À esquerda da base é colocada uma haste de metal em forma de L (seu comprimento é insuficiente para alcançar a rolha no fundo do tubo menor), e um recipiente pequeno com uma tampa de rosquear, retirada e deitada ao lado. Pede-se ao avaliado para retirar a rolha para fora do tubo menor usando qualquer um dos objetos dispostos à sua frente, sem levantar a base, os tubos e sem tocar a tampa do tubo maior com seus dedos.

3. Teste de Procurar Chaves - avalia a habilidade em planejar uma estratégia para resolver um problema. O desempenho é avaliado pelo número de estratégias criadas e o quanto estas são sistemáticas, eficientes e eficazes. É apresentado aos avaliados uma folha de papel A4 com um quadrado de $100 \mathrm{~mm}$ no meio e um pequeno ponto preto 50 milímetros abaixo dele. O indivíduo é orientado a imaginar que o quadrado é um grande campo no qual, realizando um passeio, perdeu suas chaves. A partir do ponto preto o sujeito deve desenhar uma linha mostrando o percurso que faria no campo, tendo absoluta certeza de que encontraria as chaves.

4. Teste do Julgamento Temporal - envolve julgamento e pensamento abstrato baseado no senso comum. O respondente é solicitado a estimar tempos para quatro eventos diários. O desempenho é avaliado na precisão da estimativa.

5. Teste do Mapa do Zoológico - avalia a habilidade de formular e implementar um plano, como de seguir um plano pré-formulado. Envolve o delineamento ou seguimento de uma rota por meio de um mapa. O desempenho é avaliado por meio da implementação bem-sucedida do plano. Os indivíduos são orientados a mostrar como eles visitariam uma série de locais indicados em um mapa de um zoológico, sem quebrar algumas regras deixadas em sua frente. O mapa e as regras foram construídos de modo que há apenas quatro rotas possíveis a serem tomadas, sem que as regras sejam violadas. São apresentadas duas versões do teste. Na primeira versão, o avaliado deve construir sua própria rota, mas, na segunda, apenas seguir as instruções apresentadas.

6. Teste dos Seis Elementos Modificado - avalia a habilidade em administrar o tempo. Os respondentes devem distribuir o tempo disponível (10 minutos) entre um número de tarefas simples (nomear figuras, fazer cálculos matemáticos e responder duas questões) divididas em duas partes (A e B) e obedecendo algumas regras. Devem fazer, ao menos, um pouco de cada uma das seis subtarefas nesse espaço de tempo, sem fazer as duas partes da mesma tarefa consecutivamente, isto é, responder A seguido de B da mesma subtarefa.

Questionário Disexecutivo (DEX) - questionário composto por 20 itens que descrevem comportamentos associados à SD. A avaliação da frequência desses comportamentos se dá por meio de uma escala tipo Likert, com pontuação de zero a quatro (nunca, ocasionalmente, às vezes, frequentemente, sempre), totalizando no máximo 80 pontos. Há uma versão para ser usada com o respondente e uma segunda versão para seu familiar ou cuidador que possa avaliar o respondente. Os resultados dos questionários não são utilizados no cálculo do escore total da BADS.

\section{Procedimentos}

$\mathrm{Na}$ primeira etapa, a BADS foi traduzida para o português do Brasil por três avaliadores independentes a partir de sua versão original da língua inglesa. Os tradutores foram voluntários convidados a participar da pesquisa. Estes receberam um protocolo com informações sobre o instrumento e orientações sobre o procedimento de tradução. Esse processo resultou em três versões que foram analisadas, na etapa seguinte, pelas pesquisadoras, para a formulação de uma nova versão preliminar sintetizada da BADS.

$\mathrm{Na}$ terceira etapa, a versão preliminar foi apresentada a um grupo de três juízes especialistas em neuropsicologia, objetivando minimizar possíveis vieses encontrados na tradução como divergências linguísticas, culturais, de compreensão e psicológicas, procurando manter a máxima equivalência entre a versão original e a síntese das traduções, não ignorando as particularidades inerentes à cultura brasileira. Foi fornecido aos juízes especialistas um protocolo com informações sobre a BADS, as versões inglesa e brasileira do instrumento e uma planilha para avaliação dos seguintes critérios: clareza de linguagem (CL), pertinência prática (PP), relevância teórica (RT) e dimensão teórica (DT) (Tabela 2).

A CL considera a adequação entre a linguagem dos itens do instrumento e as características da população ao qual se destina. A PP avalia a importância de cada item para o instrumento. A RT considera a relação entre o item e a teoria, ou seja, o construto, e, por fim, a DT investiga a que dimensão (fator) cada item pertence (Cassepp-Borges, Balbinotti, \& Teodoro 2010). As DTs identificadas originalmente para o instrumento foram: comportamento (A), cognição (B) e emoção (C). 
De posse destas informações os juízes deveriam fornecer a pontuação adequada em cada um dos critérios mencionados anteriormente, conforme planilha disponibilizada a eles (Tabela 2) para analisar cada subteste da BADS e itens do questionário DEX.

A partir das respostas dos juízes foi calculado o coeficiente de validade de conteúdo (CVC), que considera o número de juízes e a média de suas respostas, e o coeficiente kappa. Ambos os métodos avaliam a concordância entre os juízes, no entanto, o CVC é utilizado para variáveis contínuas e o coeficiente kappa para variáveis categóricas. Os conceitos CL, PP e RT foram utilizados para o cálculo do CVC, avaliado por meio de uma escala do tipo Likert. O ponto de corte sugerido pelo autor, considerando a aceitabilidade do item ou teste, é de 0,80 (Hernández-Nieto, 2002).

Para o critério DT, por se tratar de uma variável categórica, foi utilizado o cálculo do coeficiente kappa. Como regra, esse coeficiente é calculado para a concordância entre dois juízes. No presente estudo, porém, houve a participação de três juízes, o que levou à necessidade de utilização do cálculo do kappa médio. Para a interpretação desse coeficiente, foi utilizado o critério de Landis e Koch (1977).

$\mathrm{Na}$ quarta etapa, foi organizada uma enquete com adultos, em sua maioria alunos de graduação e pós-graduação. O subteste Julgamento Temporal foi aplicado em grupo, durante as aulas, e os alunos deveriam responder de forma espontânea, apenas anotando em uma folha de respostas o tempo que acreditavam ser necessário para que algumas coisas acontecessem.

A versão resultante constituiu a quinta etapa, apresentada em um grupo focal selecionado por conveniência. No grupo, foram apresentadas as instruções dos subtestes e questionários e levantadas as dificuldades de compreensão destas. Após ajustes no instrumento, foi realizada a sexta etapa, por meio de um estudo piloto com uma amostra de conveniência de 40 adultos que preencheram os critérios diagnósticos para a DP. Esse estudo tinha por objetivo verificar a adequação e compreensão do teste, detectando e corrigindo mais alguns pontos da BADS, resultando em uma versão final. Essa versão, após o estudo piloto, foi retrotraduzida (backtranslation) para a língua inglesa e encaminhada para avaliação da editora, finalizando a sétima etapa. Todos os participantes do estudo foram informados sobre os objetivos de estudo e, após a leitura, assinaram o Termo de Consentimento Livre Esclarecido.

\section{Análise dos Dados}

Foram utilizados os coeficientes kappa e CVC para a análise das respostas dos juízes especialistas, objetivando-se a adequação da versão traduzida da BADS. Os resultados da enquete para as quatro questões do teste Julgamento Temporal e do estudo piloto foram analisados por meio de estatística descritiva.

\section{Considerações Éticas}

Foram adotados todos os procedimentos éticos que normatizam o funcionamento de uma pesquisa. Além da aprovação pelo Comitê de do Hospital de Clínicas de Porto Alegre (CEP-HCPA), a tradução do instrumento foi autorizada pela autora.

\section{Resultados}

Para a verificação da adequação da versão traduzida unificada da BADS, avaliaram-se os escores obtidos por meio dos juízes especialistas, de acordo com os procedimentos especificados acima. A Tabela 3 mostra os resultados do CVC para cada subteste e item do questionário DEX em relação aos três critérios avaliados (CL, PP e RT). Para o critério DT, os números apresentados na tabela referem-se à frequência dos juízes, resultando no coeficiente kappa, comparado ao identificado pelos autores do instrumento.

Os itens do questionário DEX e subtestes que apresentaram CVC inferior ao ponto de corte foram reformulados de acordo com as sugestões fornecidas pelos juízes avaliadores. Após, foram enviados para

Tabela 2

Critérios de Avaliação para Juizes Avaliadores

\begin{tabular}{lccccc}
\hline $\begin{array}{l}\text { Subteste/ } \\
\text { Item }\end{array}$ & $\begin{array}{c}\text { Clareza de } \\
\text { Linguagem }\end{array}$ & $\begin{array}{c}\text { Pertinência } \\
\text { Prática }\end{array}$ & $\begin{array}{c}\text { Relevância } \\
\text { Teórica }\end{array}$ & $\begin{array}{c}\text { Dimensão } \\
\text { Teórica }\end{array}$ & Observação \\
\hline & 112 I 3 I 4 I 5 & 112 I 3 I 4 I 5 & 112 I 3 I 4 I 5 & A I B I C & \\
\hline
\end{tabular}

Nota. A: comportamento; B: cognição; C: emoção. 
Tabela 3

Cálculo do Coeficiente de Validade de Conteúdo e Avaliação da Dimensão Teórica dos Subtestes da BADS e Itens do Questionário DEX

\begin{tabular}{|c|c|c|c|c|c|c|c|c|}
\hline \multirow{2}{*}{$\mathrm{N}$} & \multirow{2}{*}{ Subteste/Item DEX } & \multicolumn{3}{|c|}{ CVC } & \multicolumn{4}{|c|}{ Dimensão Teórica } \\
\hline & & CL & $\mathrm{PP}$ & RT & $\mathrm{C}$ & $\mathrm{Co}$ & $\mathrm{E}$ & A \\
\hline 1 & Teste de Cartas de Alternar Regras & 0,89 & 0,96 & 0,96 & & 3 & & $\mathrm{C} / \mathrm{Co}$ \\
\hline 2 & Teste do Programa de Ação & 0,96 & 0,97 & 0,97 & 2 & 1 & & $\mathrm{C}$ \\
\hline 3 & Teste de Procurar Chaves & 0,82 & 0,97 & 0,97 & 1 & 2 & & $\mathrm{C}$ \\
\hline 4 & Teste de Julgamento Temporal & 0,89 & 0,97 & 0,97 & & 3 & & $\mathrm{C}$ \\
\hline 5 & Teste do Mapa do Zoológico & 0,76 & 0,97 & 0,97 & 1 & 2 & & Co \\
\hline 6 & Teste dos Seis Elementos Modificado & 0,82 & 0,97 & 0,97 & 1 & 2 & & $\mathrm{C} / \mathrm{Co}$ \\
\hline 1 & $\begin{array}{l}\text { Eu tenho problemas em entender o que as outras } \\
\text { pessoas querem dizera menos que seja algo simples e direto. }\end{array}$ & 0,83 & 0,83 & 0,83 & & 3 & & $*$ \\
\hline 2 & Eu ajo sem pensar, fazendo a primeira coisa que vem à cabeça. & 0,96 & 0,96 & 0,96 & 3 & & & $\mathrm{C}$ \\
\hline 3 & $\begin{array}{l}\text { Às vezes falo de eventos ou detalhes que na verdade nunca } \\
\text { aconteceram, mas acredito que aconteceram. }\end{array}$ & 0,90 & 0,96 & 0,96 & & 3 & & Co \\
\hline 4 & Tenho dificuldade em pensar sobre ou planejar o futuro. & 0,90 & 0,90 & 0,90 & & 3 & & $*$ \\
\hline 5 & $\begin{array}{l}\text { Às vezes fico muito animado com algumas coisas e posso } \\
\text { exagerar nestes momentos. }\end{array}$ & 0,90 & 0,96 & 0,96 & 1 & & 2 & $\mathrm{E}$ \\
\hline 6 & $\begin{array}{l}\text { Misturo alguns eventos com outros e fico confuso com a } \\
\text { ordem correta dos eventos. }\end{array}$ & 0,83 & 0,96 & 0,96 & & 3 & & $\mathrm{Co}$ \\
\hline 7 & $\begin{array}{l}\text { Tenho dificuldade em perceber a extensão dos meus } \\
\text { problemas e não sou realista sobre o futuro. }\end{array}$ & 0,96 & 0,96 & 0,96 & 1 & 2 & & $\mathrm{C}$ \\
\hline 8 & Pareço letárgico ou apático com as coisas. & 0,96 & 0,96 & 0,96 & 1 & & 2 & $\mathrm{E}$ \\
\hline 9 & $\begin{array}{l}\text { Faço ou digo coisas embaraçosas, quando na companhia de } \\
\text { outros. }\end{array}$ & 0,96 & 0,96 & 0,96 & 3 & & & $\mathrm{C}$ \\
\hline 10 & $\begin{array}{l}\text { Em um momento, quero realmente fazer algo, mas depois } \\
\text { não me importomais. }\end{array}$ & 0,96 & 0,96 & 0,96 & 1 & 1 & 1 & * \\
\hline 11 & Tenho dificuldade em demonstrar emoção. & 0,96 & 0,96 & 0,96 & & & 3 & $\mathrm{E}$ \\
\hline 12 & Perco a paciência por qualquer coisa. & 0,96 & 0,96 & 0,96 & 1 & & 2 & $\mathrm{C}$ \\
\hline 13 & $\begin{array}{l}\text { Pareço despreocupado sobre como devo me comportar em } \\
\text { determinadassituações }\end{array}$ & 0,69 & 0,96 & 0,96 & 3 & & & $\mathrm{C}$ \\
\hline 14 & $\begin{array}{l}\text { Quando começo a dizer ou fazer alguma coisa, acho difícil } \\
\text { parar derepeti-las. }\end{array}$ & 0,76 & 0,69 & 0,69 & 3 & & & Co \\
\hline 15 & $\begin{array}{l}\text { Tendo a ser muito agitado, e não consego ficar parado por } \\
\text { algum tempo. }\end{array}$ & 0,89 & 0,89 & 0,89 & 3 & & & $\mathrm{C}$ \\
\hline 16 & $\begin{array}{l}\text { Encontro difículdades para parar de fazer algo mesmo que } \\
\text { saiba que devoparar. }\end{array}$ & 0,89 & 0,96 & 0,96 & 3 & & & $\mathrm{C}$ \\
\hline 17 & Digo uma coisa, e faço outra diferente. & 0,96 & 0,96 & 0,96 & 2 & 1 & & $*$ \\
\hline 18 & $\begin{array}{l}\text { Tenho dificuldade em manter a atenção em algo, e sou } \\
\text { facilmente distraído. }\end{array}$ & 0,83 & 0,96 & 0,96 & & 3 & & Co \\
\hline 19 & Tenho problemas em tomar decisões, ou decidir o que fazer. & 0,96 & 0,96 & 0,96 & & 3 & & Co \\
\hline 20 & $\begin{array}{l}\text { Desconheço ou não me preocupo com o que os outros } \\
\text { sentem ou pensam sobre meu comportamento. }\end{array}$ & 0,89 & 0,96 & 0,96 & & 1 & 2 & $\mathrm{C}$ \\
\hline
\end{tabular}

Nota. C: comportamento; Co: cognição; E: emoção; A: dimensão teórica proposta pelos autores da BADS; CVC: coeficiente de validade de conteúdo; CL: clareza de linguagem: PP: pertinência prática; RT: relevância teórica; BADS: Behavioural Assessment of Dysexecutive Syndrome; DEX: questionário disexecutivo.

* As dimensões teóricas nos itens 1,4, 10 e 17 não foram indicadas pelos autores. 
reavaliação, resultando em aumento do CVC em todos os critérios.

Observou-se que a grande maioria dos itens e subtestes apresentaram $\mathrm{CVC}>0,8$ considerados satisfatórios. A dimensão CL foi a que apresentou o maior número de itens (13 e 14) e um subteste (Teste do Mapa do Zoológico) com CVC abaixo de 0,8 e, portanto, foram reformulados e encaminhados para reavaliação pelos mesmos juízes (Tabela 4). Nos três casos houve aumento no valor do CVC de uma versão para outra. Apenas o item 14 apresentou pontuação inferior ao ponto de corte nos critérios PP e RT. Ambos os critérios também apresentaram aumento do CVC após a reformulação da tradução. Após essa etapa, realizou-se o cálculo do CVC total para a BADS e o DEX, indicando valores aceitáveis em todas as dimensões (Tabela 5).

Em relação à DT, a primeira avaliação apontou para a multidimensionalidade de alguns itens e subtestes do instrumento. No entanto, a avaliação dos dados em mais de uma dimensão é muito complexa, devendo ser evitada (Cassep-Borges et al., 2010), então, solicitou-se que cada juiz optasse por apenas uma dimensão.

Apenas dois subtestes (Procurar Chaves e Julgamento Temporal) e quatro itens do questionário DEX $(7,12,14$ e 20$)$ obtiveram número inferior a dois juízes concordando com a dimensão original proposta por Wilson, Alderman, Burgess, Emslie e Evans (1996). A média do coeficiente kappa para os subtestes da BADS foi de 0,55 e para o questionário DEX foi de 0,75 , considerados moderado e sólido, respectivamente (Landis \& Koch, 1977).

Os resultados para a enquete do Teste de Julgamento Temporal indicaram a necessidade de não adesão à resposta original apenas para a questão quatro. As respostas às questões um, dois e três foram mantidas (Tabela 6).
Para o critério CL, alguns itens e subtestes, mesmo apresentando $\mathrm{CVC}>0,8$ no critério, foram alterados tendo como base as sugestões dos juízes e membros do grupo focal e observações realizadas durante a aplicação da BADS no estudo piloto. Para as novas versões não foram calculados o CVC.

A partir da aplicação da BADS nos participantes do grupo focal e estudo piloto, foi identificada a necessidade de alteração em algumas instruções dos subtestes e itens do questionário DEX, pois se observou a dificuldade para o entendimento e execução da atividade requerida. Assim, as instruções dos seis subtestes e os itens cinco e 20 do questionário DEX sofreram parciais modificações.

\section{Discussão}

É consenso entre psicometristas a importância da adaptação de instrumentos para outras culturas, no entanto, muitos pesquisadores da área não submetem seus instrumentos aos devidos procedimentos de tradução e adaptação. Muitas vezes, são utilizadas apenas a tradução simples e direta dos itens que compõem o instrumento, analisando apenas a equivalência semântica

Tabela 5

Cálculo do Coeficiente de Validade de Conteúdo Total da $B A D S$ e DEX

\begin{tabular}{llll}
\hline Subteste & CL & PP & RT \\
\hline BADS & 0,89 & 0,96 & 0,96 \\
Questionário DEX & 0,92 & 0,94 & 0,94 \\
\hline
\end{tabular}

Nota. BADS: Behavioural Assessment of Dysexecutive Syndrome; DEX: questionário disexecutivo; CL: clareza de linguagem; PP: pertinência prática; RT: relevância teórica.

Tabela 4

Resultados da Segunda Avaliação da BADS pelos Juízes

\begin{tabular}{llccc}
\hline \multirow{2}{*}{$\mathrm{N}^{\mathrm{O}}$} & Subteste/Item DEX & \multicolumn{3}{c}{ CVC } \\
\cline { 3 - 4 } & & $\mathrm{CL}$ & $\mathrm{PP}$ & $\mathrm{RT}$ \\
\hline 5 & Teste do Mapa do Zoológico & 0,96 & 0,96 & 0,96 \\
13 & Sou indiferente sobre como devo me comportar em determinadas situações. & 0,96 & 0,96 & 0,96 \\
14 & Acho difícil parar de dizer ou fazer coisas repetidamente depois que começo. & 0,89 & 0,89 & 0,89 \\
\hline
\end{tabular}

Nota. BADS: Behavioural Assessment of the Dysexecutive Syndrome; DEX: questionário disexecutivo; CVC: coeficiente de validade de conteúdo; CL: clareze de linguagem, PP: pertinência prática, RT: relevância teórica. 
Tabela 6

Médias e Desvios-Padrão para as Respostas às Questões do Teste Julgamento Temporal

\begin{tabular}{lcccc}
\hline & Questão 1 & Questão 2 & Questão 3 & Questão 4 \\
\hline Média & 3,08 & 12,15 & 12,05 & 56,27 \\
desvio-padrão & 1,64 & 6,89 & 2,83 & 34,15 \\
\hline
\end{tabular}

entre a versão adaptada e a versão original, o que não garante a confiabilidade da medida (Hambleton, 2005; Cassepp-Borges et al., 2010). Além disto, não há consenso entre autores sobre a melhor forma de adaptar um instrumento para outro contexto cultural. Esse procedimento dependerá de distintas questões entre as quais as características do instrumento e o público a que se destina (Borsa, Damásio, \& Bandeira, 2012).

Dessa forma, o presente estudo considerou em seu processo de adaptação, a pertinência dos conceitos e domínios do instrumento original na nova cultura e a adequação destes à nova população-alvo. Além disso, considerou-se a equivalência semântica, linguística e contextual entre os itens do instrumento original e do traduzido. Tais procedimentos buscaram garantir que os resultados obtidos com o instrumento se devam a diferenças ou similaridades entre os grupos em que a SD esteja sendo mensurado e não a erros de tradução.

Sendo assim, o objetivo deste estudo foi descrever os procedimentos metodológicos de tradução e de adaptação da BADS para o português brasileiro. Para esse fim, seguiram-se os procedimentos sugeridos por diferentes autores da área, encontrando-se o CVC, coeficiente kappa, estatísticas descritivas e indicações semântico-linguísticas que nortearam as mudanças necessárias até a versão final do instrumento (Beaton, Bombardier, Guillemin, \& Ferraz 2000; Hambleton, 2005; Gjersing, Caplehorn, \& Clausen, 2010).

Uma vez que o cálculo do CVC é uma medida baseada na opinião de juízes, seus resultados devem ser tomados apenas como medidas norteadoras. Dessa forma, alguns itens e subtestes, mesmo tendo apresentado CVC satisfatório, foram alterados, baseando-se nas sugestões de juízes, participantes do grupo focal e estudo piloto. As alterações realizadas visaram a melhor compreensão e execução dos itens e subtestes, uma vez que, especialmente, pacientes com DP encontravam dificuldades em partes do instrumento, sugerindo que portadores da SD apresentam mais dificuldade, necessitando maior simplicidade nas informações.

Não foram encontrados estudos de adaptação e validade de conteúdo da BADS para outras línguas, no entanto diversas pesquisas têm demonstrado boa validade concorrente do instrumento na avaliação de diversas populações neurológicas e psiquiátricas (Bennet, Ong, \& Ponsford, 2005; Evans, Chua, Mckenna, \& Wilson, 1997; Katz et al., 2007; Norris \& Tate, 2000; Wilson et al., 1998). Outros estudos têm demonstrado que a BADS possui maior poder discriminativo em predizer alterações executivas na vida diária (Burgess et al., 2000; Chaytor \& Schmitter-Edgecombe, 2003; Chamberlain, 2003). Pesquisa realizada por Verdejo-García e Perez-García (2007) comparando a BADS ao WCST, demonstrou que aquela foi superior para detectar alterações executivas.

No Brasil, apenas três estudos foram encontrados utilizando a BADS, demonstrando a necessidade de pesquisas que busquem evidências de validade desse instrumento em amostras distintas. $O$ estudo de Canali, Brucki e Bueno (2007) utilizou a BADS, para avaliar idosos saudáveis e pacientes com doença de Alzheimer (DA) em fase inicial. O instrumento foi eficiente na detecção de prejuízos executivos na amostra, confirmando os resultados obtidos pelos demais instrumentos de avaliação de FE utilizados. Em outro estudo, comparou-se o desempenho da BADS em uma amostra de indivíduos saudáveis, com comprometimento cognitivo leve (CCL) e DA. Foram encontradas diferenças significativas no desempenho da BADS entre os indivíduos saudáveis e o grupo de pacientes com DA. A BADS também se mostrou sensível para discriminar diferenças no desempenho entre DA e CCL (Armentano, Porto, Brucki, \& Nitrini, 2009). O terceiro avaliou idosos saudáveis e pacientes com DA em um estudo de confiabilidade da BADS, que se mostrou eficaz na detecção dos déficits em FE entre os pacientes, especialmente nas tarefas de alternância, monitoramento de tempo e subtestes de mudanças de regras (Canali, Brucki, Bertolucci, \& Bueno, 2011).

Recentemente, dois estudos forneceram dados sobre as vantagens de se usar a BADS em relação a outras medidas em pacientes com DP. O estudo de Kamei et al. (2008) demonstrou que a pontuação em 
todos os subtestes da BADS, em pacientes com disfunção executiva, foram significativamente inferiores aos dos pacientes sem disfunção executiva. Por fim, o estudo de Perfetti et al. (2010) evidenciou que a BADS é mais sensível para avaliar disfunções executivas do que alguns dos instrumentos tradicionalmente usados para avaliar esse traço na DP.

Dessa forma, os bons resultados obtidos em diferentes pesquisas com a BADS e a ausência de uma adaptação meticulosa para o Brasil, reforçam a relevância do presente estudo. Após os procedimentos de tradução e adaptação da BADS, concluiu-se que o instrumento está apto para dar seguimento aos estudos de fidedignidade, validação de construto, validação clínica, bem como estudo de normatização. Reconhecem-se as limitações do estudo, principalmente no que se refere ao grupo clínico específico de pacientes portadores da DP. Destaca-se ainda o caráter ecológico da bateria e os benefícios dessa abordagem.

\section{Referências}

Armentano, C. G. da C., Porto, C. S., Brucki, S. M. D., \& Nitrini, R. (2009). Study on the Behavioural assessment of the dysexecutive syndrome (BADS) performance in the healthy individuals, mild cognitive impairment and Alzheimer's disease. A preliminary study. Dementia \& Neuropsychologia, 3(2), 101-107. Recuperado de http://www. demneuropsy.com.br/imageBank/PDF/dnv03n02a05.pdf

Baddeley, A. D., \& Hitch, G. (1974). Working memory. Em G. H. Bower (Ed.), The psychology of learning and motivation (p. 47-89). New York: Academic Press.

Barkley, R. (2001). The executive functions and selfregulation: An evolutionary neuropsychological perspective. Neuropsychology Review, 11(1), 1-29. doi: 10.1023/A:1009085417776

Beaton, D. E., Bombardier, C., Guillemin, F., \& Ferraz, M. B. (2000). Guidelines for the process of crosscultural adaptation of self-report measures. Spine, 24, 3186-3191. Recuperado de http://www.ncbi. nlm.nih.gov/pubmed/11124735

Bennett, P. C., Ong, B., \& Ponsford, J. (2005). Assessment of executive dysfunction following traumatic brain injury: Comparison of the BADS with oder clinical neuropsychological measures. Journal of the International Neuropsychological Society,
11(5), 606-613. Recuperado de http://www.ncbi. nlm.nih.gov/pubmed/16212688

Borges, M., Coutinho, G., Miele, F., Malloy-Diniz, L. F., Martins, R., Rabelo, B., \& Mattos, P. (2010). Síndromes disexecutivas do desenvolvimento e adquiridas na prática clínica: Três relatos de caso. Revista Psiquiatria Clinica, 37(6), 285-290. Recuperado de http://www.scielo.br/pdf/rpc/v37n6/ a07v37n6

Borsa, J. C., Damásio, B. F., \& Bandeira, D. R. (2012). Adaptação e validação de instrumentos psicológicos entre culturas: Algumas considerações. Paidéia, 22(53), 423-432. doi: 10.1590/1982-43272253201314

Burgess, P. W., Costello, A., \& Shallice, T. (2000). The cognitive and neuroanatomical correlates of multitasking. Neuropsychologia, 38, 848-863. doi:10.1016/S0028-3932(99)00134-7

Campos-Sousa, I. S., Campos-Sousa, R. N., Ataíde Jr., L., Soares, M. M. de B., \& Almeida, K. J. (2010). Executive dysfunction and motor symptoms in Parkinson's disease. Arquivos de Neuropsiquiatria, 68(2), 246-251. Recuperado de http://www.scielo. $\mathrm{br} / \mathrm{pdf} / \mathrm{anp} / \mathrm{v} 68 \mathrm{n} 2 / 18 . \mathrm{pdf}$

Canali, F., Brucki, S. M. D., \& Bueno, O. F. A. (2007). Behavioural assessment of the dysexecutive syndrome (BADS) in healthy elders and Alzheimer's disease patients. Preliminary study. Dementia \& Neuropsychologia, 2, 154-160. Recuperado de https:// www.researchgate.net/publication/233884337_ Behavioural_Assessment_of_the_Dysexecutive_ Syndrome_for_Children_BADS-C

Canali, F., Brucki, S. M. D., Bertolucci, P. H. F., \& Bueno, O. F. A. (2011). Reliability study of the Behavioral assessment of the dysexecutive syndrome adapted for a Brazilian sample of older-adult controls and probable early Alzheimer's disease patients. Revista Brasileira de Psiquiatria, 33(4), 338-346. Recuperado de http://www.ncbi.nlm.nih.gov/ pubmed/21584345

Cassepp-Borges, V., Balbinotti, M. A. A., \& Teodoro, M. L. M. (2010). Tradução e validação de conteúdo: Uma proposta para a adaptação de instrumentos. Em L. Pasquali. (Ed.), Instrumentação Psicológica: fundamentos e práticas. ( $1^{\mathrm{a}}$ ed.). Porto Alegre: Artmed.

Chamberlain, E. (2003). Test review - Behavioural assessment of the dysexecutive syndrome 
(BADS). Journal of Occupational Psychology, Employment and Disability, 5(2), 33-37. doi: 10.1080/09297040601112781

Chaytor, N., \& Schmitter-Edgecombe, M. (2003). The ecological validity of neuropsychological tests: a review of the literature on everyday cognitive skills. Neuropsychology Review, 13(4), 181-197. doi: 10.1023/B:NERV.0000009483.91468.fb

Evans, J. J., Chua, S. E., McKenna, P. J., \& Wilson, B. A. (1997). Assessment of the dysexecutive syndrome in schizophrenia. Psychological Medicine, 27, 635-646. Recuperado de http://hub.hku.hk/ bitstream/10722/43485/1/25229.pdf

Fuentes, D., Malloy-Diniz, L. F., Camargo, C. H. P. de, \& Consenza, R. M. (2014). Neuropsicologia: teoria e prática. (2a ed.). Porto Alegre: Artmed.

Galhardo, M. M. de A. M. C., Amaral, A. K. F. J. do, \& Vieira, A. C. C. (2009). Caracterização dos distúrbios cognitivos na doença de Parkinson. Revista Cefac, 11(2), 251-257. Recuperado de http://www. scielo.br/pdf/rcefac/v11s2/a15v11s2.pdf

Geisinger, K. F. (1994). Cross-cultural normative assessment: Translation and apadtation issues influencing the normative interpretation of assessments instruments. Psychological Assessment, 6, 304-312.

Gjersing, L., Caplehorn, J. R. M., \& Clausen, T. (2010). Cross-cultural adaptation of research instruments: Language, setting, time and statistical considerations. BMC Medical Research Methodology, 10(13). doi: 10.1186/1471-2288-10-13

Godefroy, O., Garcia, P. Y., Bugnicourt, J. M., Leclercq, C., \& Roussel, M. (2014). The behavioral consequences of stroke. Em T. A. Schweizer \& R. L. Macdonald (Eds.). Dysexecutive syndrome after stroke(pp. 135-150). New York: Springer.

Hambleton, R. K. (2005). Issues, designs, and technical guidelines for adapting tests into multiple languages and cultures. Em R. K. Hambleton, P. F. Merenda \& C. D. Spielberger (Eds.), Adapting educational and psychological tests for cross-cultural assessment(pp. 3-38). Mahwah, NJ: Lawrence Erlbaum Associates.

Hamdan, A. C., \& Pereira, A. P. de A. (2009). Avaliação neuropsicológica das funções executivas: Considerações metodológicas. Psicologia: Reflexão e Crítica,
22(3), 386-393. Recuperado de http://www.scielo. $\mathrm{br} / \mathrm{pdf} / \mathrm{prc} / \mathrm{v} 22 \mathrm{n} 3 / \mathrm{v} 22 \mathrm{n} 3 \mathrm{a} 09 . \mathrm{pdf}$

Hernández-Nieto, R. A. (2002). Contribuciones al análisis estadístico. Mérida, Venezuela: Universidad de Los Andes.

Imamura, K., Wada-Isoe, K., Kitayama, M., \& Nakashima, K. (2008). Executive dysfunction in non-demented Parkinson's disease patients with hallucinations. Acta Neurologica Scandinavica, 117, 255-259. doi: 10.1111/j.1600-0404.2007.00933.x

Kamei, S., Hara, M., Serizawa, K., Murakami, M., Mizutani, T., Ishiburo, M., Kawahara, R., Takagi, Y., Ogawa, K., Yoshihashi, H., Shinbo, S., Suzuki, Y., Yamaguchi, M., Morita, A., Takeshita, J., \& Hirayanagi, K. (2008). Executive dysfunction using behavioral assessment of the dysexecutive syndrome in parkinson's disease. Movement Disorders, 23(4), 566-573. Recuperado de ncbi.nlm.nih.gov/ pubmed/18098279

Kamei, S., Morita, A., Serizawa, K., Mizutani, T., \& Hirayanagi, K. (2010). Journal of Clinical Neurophysiology, 27(3), 193-197.

Katz, N., Tadmor, I., Felzen, B., \& Hartman-Maeir, A. (2007). The Behavioral Assessment of the Dysexecutive Syndrome (BADS) in schizophrenia and its relation to functional outcomes. Neuropsychological Rehabilitation, 22,1-14. Recuperado de http:// www.ncbi.nlm.nih.gov/pubmed/17454693

Kummer, A., \& Teixeira, A. L. (2009). Neuropsychiatry of Parkinson's disease. Arquivos de Neuro-Psiquiatria, 67(3b), 930-939. Recuperado de http:// www.ncbi.nlm.nih.gov/pubmed/15550293

Landis, J. R., \& Koch, G. G. (1977). The measurement of observer agreement for categorical data. Biometrics, 33, 159-174.

Malloy-Diniz, L. F., Paula, J. J. de, Loschiavo-Alvares, F. Q., Fuentes, D., \& Leite, W. B. (2010). Exame das funções executivas. Em L. F. Malloy-Diniz, D. Fuentes, \& P. Mattos, Avaliação neuropsicológica. Porto Alegre: Artmed.

Malloy-Diniz, L. F., Sedo, M., Fuentes, D., \& Leite, W. B. (2008). Neuropsicologia das funções executivas. Em D. Fuentes, L. F. Malloy-Diniz, C. H. P. Camargo \& R. M. Cosenza (Eds.). Neuropsicologia: teoria e prática (pp. 187-206). Porto Alegre: Artmed. 
Mckinlay, A., Grace, R. C., Dalrymple-Alford, J. A., \& Roger, D. (2010). Characteristics of executive function impairment in Parkinson's disease patients without dementia. Journal of the International Neuropsychological Society, 16, 268-277. doi: 10.1017/S1355617709991299

Montiel, J. M., \& Seabra A. G. (2012). Teste de trilhas partes A e B (pp. 79-85). Em A. G. Seabra \& N. M. Dias (Eds.). Avaliação neuropsicológica cognitiva: atenção e funcões executivas. São Paulo: Memnon.

Norris, G., \& Tate, R. L. (2000). The behavioural assessment of the dysexecutive syndrome (BADS): Ecological, concurrent and construct validity. Neuropsychological Rehabilitation, 10, 33-45. doi:10.1080/096020100389282

Parsons, T. D. (2011). Neuropsychological assessment using virtual environments: enhanced assessment technology for improved ecological validity. Em S. Brahnam \& L. C. Jain, Advanced computational intelligence paradigms in healthcare 6. Virtual reality in psychotherapy, rehabilitation, and assessment studies in computational intelligence (271-289). New York: Springer.

Pasquali, L. (2001). Parâmetros psicométricos dos testes psicológicos. Em Pasquali, L. (Ed.). Técnicas de exame psicológico - TEP: manual, v. 1. São Paulo: Casa do Psicólogo/CFP.

Perfetti, B., Varanese, S., Mercuri, P., Mancino, E., Saggino, A., \& Onofrj, M. (2010). Behavioural assessment of dysexecutive syndrome in Parkinson's disease without dementia: a comparison with other clinical executive tasks. Parkinsonism \& Related Disorders, 16(1), 46-50. doi:10.1016/j. parkreldis.2009.07.011

Sammer, G., Reuter, I., Hullmann, K., Kaps, M., \& Vaitl, D. (2006). Training of executive functions in Parkinson's disease. Journal of the Neurological
Sciences, 248, 115-119. Recuperado de http:// www.ncbi.nlm.nih.gov/pubmed/16765378

Sandoval, J., \& Durán, R. P. (1998). Language. Em J. Sandoval, C. L. Frisby, K. F. Geisinger, J. D., Sheuneman \& J. R. Grenier (Eds.), Test interpretation and diversity: Achieving equity in assessment wording. Washington, DC: American Psychological Association.

Silva, F. C. da, Macedo, L. F. R., Mariani, M. M. de C., Araújo, R. R. de, \& Carreiro, L. R. R. (2011). Efeitos do controle de previsibilidade e da utilização de pistas simbólicas centrais na orientação temporal da atenção. Psicologia Teoria e Prática, 13(3). Recuperado de http://pepsic.bvsalud.org/pdf/ptp/ v13n3/v13n3a03.pdf

Sternberg, R. J. (2000). Psicologia cognitiva. Porto Alegre: Artmed.

Strauss, E., Sherman, E. M. S., \& Spreen, O. (2006). A compendium of neuropsychological tests: administration, norms, and commentary. Oxford: Oxford University Press.

Verdejo-García, A., \& Miguel Pérez-García (2007). Drug and Alcohol Dependence, 90(1), 48-55.

Wilson, B. A., Alderman, N., Burgess, P. W., Emslie, H., \& Evans, J. J. (1996). The behavioural assessment of the dysexecutive syndrome .Bury St Edmunds: Thames Valley Company.

Wilson, B. A., Evans, J. J., Emslie, H., Alderman, N., \& Burgess, P. (1998). The development of an ecologically valid test for assessing patients with dysexecutive syndrome. Neuropsychological Rehabilitation, 8, 213-228. doi: 10.1080/713755570

Recebido em: 12/06/2014

Reformulado em: 24/05/2015

Aprovado em: 13/07/2015 
Nota das autoras:

Apoio: Capes.

Sobre as autoras:

Greici Rössler Macuglia é graduada em Psicologia pela Universidade Federal de Santa Maria (2004), mestre e doutoranda em Psicologia pela Universidade Federal do Rio Grande do Sul (CAPES 7) e especialista em Psicologia Hospitalar pelo Conselho Federal de Psicologia.

E-mail:gmacuglia@uol.com.br

Rosa Maria Martins de Almeida possui graduação, mestrado e doutorado em Psicologia pela Universidade Federal do Rio Grande do Sul (1990/1992/1997), pós-doutora pela Tufts University - USA (2002) e professora adjunta da Universidade Federal do Rio Grande do Sul (UFRGS).

E-mail:rosa_almeida@yahoo.com

Fabiane Caillava Santos é graduada em Psicologia pela Universidade Federal de Santa Maria (2004), especialista em Neuropsicologia pela Universidade Luterana do Brasil (2005) e membro da Sociedade Brasileira de Neuropsicologia. E-mail:fabianecaillava@hotmail.com

Cláudia Hofheinz Giacomoni é psicóloga pela Pontifícia Universidade Católica do Rio Grande do Sul (1995), mestre e doutora em Psicologia pela Universidade Federal do Rio Grande do Sul (1997/2002) e professora do Programa de Pós-graduação em Psicologia da Universidade Federal do Rio Grande do Sul.

E-mail: giacomonich@gmail.com

Contato com as autoras:

Greici Rössler Macuglia

Universidade Federal do Rio Grande do Sul, Instituto de Psicologia

Rua Ramiro Barcelos, 2600, sala 105. Santa Cecília

CEP: 90035-003

Porto Alegre-RS, Brasil

Psico-USF, Bragança Paulista, v. 21, n. 2, p. 219-231, mai./ago. 2016 


\section{ERRATA}

No artigo Behavioral Assessment of the Dysexecutive Syndrome (BADS): Adaptation and Validity Evidence, com número de DOI: <10.1590/1413-82712016210201>, publicado no periódico Psico-USF, 21(2): páginas 219-231, na página 219 onde se lia:

'Fabiane Caillava Santos Silvia Helena Koller'

leia-se:

'Fabiane Caillava Santos' 